\title{
Communication in the Digital Work Environment: Implications for Wellbeing at Work'
}

I Laura Bordi

Researcher, University of Tampere, Faculty of Management, Finland

I Jussi Okkonen

Senior Researcher, University of Tampere, Faculty of Communication Sciences, Finland

I Jaana-Piia Mäkiniemi

Postdoctoral Researcher, University of Tampere, Faculty of Management, Finland

I Kirsi Heikkilä-Tammi

Research Director, University of Tampere, Faculty of Management, Finland

\begin{abstract}
This article examines digital communication in the workplace and its association with wellbeing at work. The analysis is based mainly on workshop discussions and is complemented with log data $(N=36)$. Content analysis was applied to the workshop discussions, while the log data were analyzed by quantifying frequencies. Six themes were found to affect wellbeing at work: the volume of digital communication, expectations of constant connectivity, the quality of the messages, adaptation of new tools, technical problems, and flexibility in communication. In relation to wellbeing at work, digital communication was mostly perceived as demanding. However, some of the factors perceived as demanding could also provide flexibility, which was seen as enhancing wellbeing by increasing autonomy and control. Social factors, such as work habits, practices, and conventions in the workplace, seem to play an important role in the manifestation and management of the digital communication-induced load at work.
\end{abstract}

\section{KEYWORDS}

Communication technology / digital work environment / information ergonomics / technostress / wellbeing at work

\section{Introduction}

oday's knowledge work is characterized by digital communication and constant connectivity, which may have various implications for wellbeing at work. Digital tools can, for example, provide flexibility and autonomy but also lead to an increase in job demands and unclear boundaries between work and nonwork life (Hoonakker, 2014). A major part of the workday can be spent in asynchronous communication, which causes

\footnotetext{
${ }^{1}$ You can find this text and its DOI at https://tidsskrift.dk/njwls/index.

2E-mail: laura.bordi@uta.fi.
} 
task fragmentation (Wajcman \& Rose, 2011). Previous research has shown that even in asynchronous communication, there is often normative pressure to react immediately, which may manifest, for example, in repeated communication activities or in the sending of a copy of a message to other people to pressure the recipient to reply quickly (Barley et al., 2011). This kind of telepressure has been associated with stress-related effects, such as burnout, sleep problems, and health-related absenteeism (Barber \& Santuzzi, 2015). Asynchronous communication and telepressure also often increase spillover from work to free time. To manage work-related matters, employees often check email at home (Barley et al., 2011). This behavior has a negative effect on recovery and can cause work-life conflict (Barber \& Santuzzi, 2015; Diaz et al., 2012). In addition, the large amount and poor quality of email have been associated with emotional exhaustion, especially if the workplace exerts normative pressure to reply immediately (Brown et al., 2014).

The aim of the current study is to deepen the understanding of associations between digital communication and wellbeing at work by examining which factors in digital communication affect employee wellbeing and how. In addition, we aim to provide a general view on the digital communication environment in which employees operate.

In this study, communication refers to information-related actions involving either one-to-one or one-to-many communication. These modes underscore that one worker can simultaneously contact several others without additional effort. This effortlessness can be a blessing and a curse. The term digital communication environment refers to the tools and modes of communication. Tools refer to the means of communication, for example, instant messaging (IM), email, social media, and bulletin boards. Modes of communication refer to two aspects, spatiality and synchronicity. Communication can be local and synchronous, local and asynchronous, or spatially dispersed and asynchronous. These modes and tools can have different implications for employee wellbeing.

There are various definitions of wellbeing at work depending on viewpoint and discipline. Most studies approach the concept through positive and/or negative outcomes, such as job satisfaction, work engagement, stress, and burnout (c.f. Orsila et al., 2011). In this study, wellbeing at work is understood broadly, as being determined by various aspects of work, which may function as demands or resources in various ways. Wellbeing is understood to form in the interplay of these resources and demands (c.f. Schaufeli $\&$ Bakker, 2004). This study focuses on the impact of digital communication on wellbeing at work. Digital communication is viewed through the lens of information ergonomics, which aims at increasing wellbeing and productivity through reducing information- and technology-related loads and increasing the suitability of technology for workers and work tasks. Information ergonomics covers a wide array of organizational factors related to technology use, such as technology itself, infrastructure, social aspects, and individual habits. In this study, wellbeing at work is approached as the participants' subjective experiences of wellbeing in regard to work-related digital communication.

\section{Digital communication as a job demand and a resource}

We suggest that digital communication may act as a job demand and as a resource (c.f. Day et al., 2010). Job resources are aspects of the job that motivate, inspire, and assist in achieving objectives, whereas demands are aspects that require extra effort and exertion (Bakker \& Demerouti, 2007). Simply put, resources are associated with wellbeing at 
work, such as work engagement, which is an active, positive, work-related state characterized by vigor, dedication, and absorption, and demands are associated with ill-being, such as job stress and burnout (Schaufeli \& Bakker, 2004). However, critical approaches on the job demands-resources model suggest that the relationship between job demands and resources is more complex. For example, although resources are often perceived positively, they might also be experienced as threatening (e.g., performance feedback). In addition, not all demands are associated with ill-being, and therefore, it is important to distinguish between challenging and hindering demands. Typically, hindering demands are perceived as negative and stressful, and therefore, more threatening to wellbeing (Schaufeli \& Taris, 2014).

More precisely, when digital communication acts as a job resource, it can, for instance, support effective information and communication transfer, facilitate interaction between workers, and provide flexibility in location (Day et al., 2010; Demerouti et al., 2014). Digital communication can be a job demand if it requires extra effort, creates pressure, and disturbs workflow. In addition, the constant flow of email can increase employees' workloads and lead employees to lose control over their work (Barley et al., 2011). Asynchronous communication often promotes a need for multitasking and increases interruptions and stress (Mark et al., 2012). Multitasking involves not only switching between tasks but also switching between communication channels and communication partners (Su \& Mark, 2008). In line with the notion that job demands in general are associated with ill-being (Schaufeli \& Bakker, 2004), we assume that digital communication, when experienced as a hindering demand, may be associated with technostress. Technostress is a specific type of work stress, with symptoms such as high levels of anxiety, fatigue, skepticism, and inefficacy associated with technology use (Salanova et al., 2014). On the basis of previous findings, it seems that in the context of digital communication, technostress symptoms may appear, for example, when employees are forced to use digital communication channels although they prefer other channels, there are expectations of constant availability, communication technology is difficult to use, and technologies are constantly changing (c.f. Tarafdar et al., 2011). Taken together, using communication technologies at work may have various implications for wellbeing, depending on whether digital communication acts as a positive resource or a challenging or hindering demand.

\section{Information ergonomics as an approach to managing information load and technostress}

Information ergonomics is an emerging domain of ergonomics focusing on the management of workload and the handling of technostress in information-intensive tasks. Thus, information ergonomics can be seen as an approach to support performance and wellbeing at work in information-heavy work settings. The concept of information ergonomics emerged in the 1960s from industrial engineering and logistics. Several studies (Eppler \& Mengis, 2004; Franssila et al., 2015; Mark et al., 2014; Wajcman \& Rose, 2011) have since reported the benefits of an information environment tailored to the individual, rather than the reverse. Franssila et al. (2015) provided the first Nordic attempt to apply the concept in a knowledge work context and to take the digital work environment into account.

Information ergonomics is a multifaceted phenomenon (Okkonen et al., 2017). Technology-related issues of information ergonomics consider interaction with different 
Figure I: Components and perspectives of information ergonomics (Okkonen et al., 20 I7).

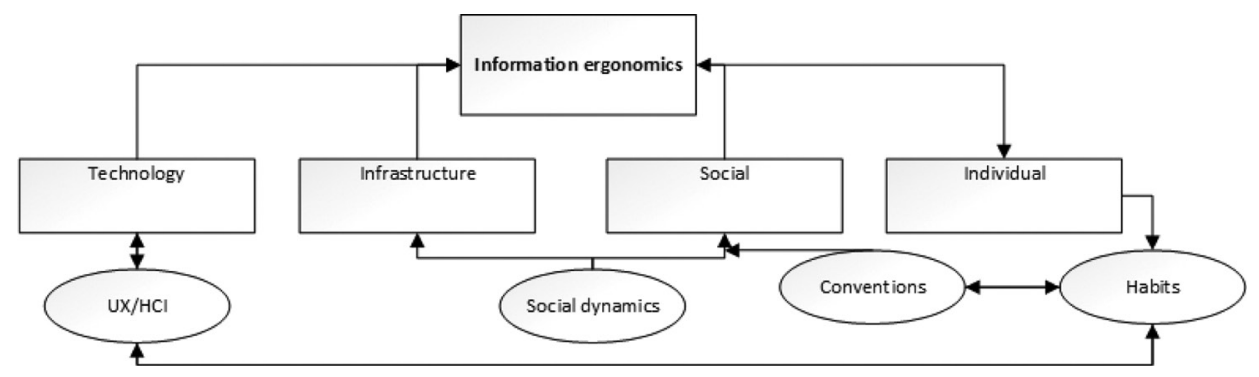

digital tools, systems, and information and communication channels. As depicted in Fig. 1, conventions, habits, and individual skills affect how one positions oneself in the digital environment and how the digital environment affects work performance. Moreover, the role of technology in this context is dominant, as technology defines the framework and functional boundaries. The infrastructure-related issues of information ergonomics consider the socio-technological system to be somewhat manageable through applications and instructions. The infrastructure consists of the physical and digital environments, hardware and software, and intentions about the purposeful use of these assets. Infrastructure affects ergonomics directly as technological restraint and through social factors. The social factors of information ergonomics have a vicarious effect, as they are the products of interplay between individuals in the digital environment. Social factors can be seen as socially constructed conventions and sets of explicit and implicit contracts. Social factors and infrastructure are closely related, but a distinction should be made. Individual factors of information ergonomics are the most obscure elements, and they are also variable. The (micro)actions and decisions of individuals during their active hours are dependent on the nature of the task and the work environment. As knowledge work involves mostly managing and analyzing information, the most significant factor is the digital work environment.

Based on the analysis above, information ergonomics refers to how a user interacts with the digital environment and is affected by the digital environment. Skills and habits are crucial factors in the user experience; thus, user experience issues explain the functionality of the human-computer apparatus. As knowledge work involves gathering, analyzing, and disseminating information and knowledge, the digital work environment is also a tool for cooperation and communication. Communication is a significant information ergonomic factor in the digital work environment. As this paper addresses communication as an explanatory factor of work performance and wellbeing at work, the information ergonomics approach is well suited as an analysis framework for the digital work realm. As explained in Fig. 1, communication-related issues are visible at the organizational level, that is, technology, infrastructure, social, and individual. However, communication also affects user experience, social dynamics, conventions, and habits. Several work tasks are communication-related, and therefore, information ergonomics is a suitable lens.

The research questions we aim to answer in this article are as follows: How is communication in the digital work environment associated with wellbeing at work? Which factors in digital communication affect wellbeing at work, and how? In what kind of digital communication environment are employees operating? 


\section{Methods}

\section{Study design}

The current study was part of a larger research project $(n=36)$ focusing on information ergonomics. The project was mixed-method action research, which included questionnaires, psychophysiological measures [heart rate variability (HRV)], the collection of log data, and workshop discussions. As is characteristic of action research, the participants were not only informants but also active participants in the research process (Somekh, 2006). The empirical data used for this article consist only of the log data and workshop discussions. However, participants were provided with the results of the questionnaires and measures, which they could utilize as background information for the workshops. The questionnaires covered the organizations' general state of employee wellbeing and information load, while the combined results of the log and HRV data provided information on how activities in the digital work environment affect employees' stress levels. These data provided useful background information for the workshops, the ultimate goal of which was to discuss issues regarding the digital work environment and wellbeing at work and develop ways to enhance information ergonomics in the workplace. Digital communication was one of the main recurring topics in the workshops, which is why it was chosen as a topic for this study. The project was carried out and the data were gathered between May 2015 and May 2016.

The novelty of the current study design stems from the fact that previous research on digital communication has often been quantitative, focused on a single communication tool (especially email) or a single outcome of the use of communication technology (e.g., multitasking). However, little is known about how a workplace's digital communication landscape operates as a whole and its implications for wellbeing at work. In this study, the log data were used to provide a general view of digital communication (e.g., tools used and time spent on digital communication), which provides a background for the employees' insights into digital communication and its effects on wellbeing at work provided during the workshop discussions.

\section{Participants}

A total of 36 participants represented three organizations: an industrial company $(n=13)$, an insurance company $(n=13)$, and a financial administration services company $(n=10)$. The organizations had been contacted by the researchers and invited to participate in the study. Because the research project dealt with work in digital work environments and information ergonomics, the criterion for participation was that the employees were, by broad definition, knowledge workers. The participating employees were recruited through contact persons in the organizations. Participants signed up voluntarily with the contact person, and the contact person provided the final participant list to the researchers. Before the study, the participants were provided with further information and asked to give consent.

Thirty-four of the participants were women. All participants in the financial administration services company were women, while the insurance company and the industrial company each had one male participant. The average age of the participants was 42.5 years. The industrial company participants mostly worked in financial 
administration and human resources. The insurance company participants were mostly insurance claims handlers or customer advisors. In the financial administration services company, most of the participants worked in payroll. In each organization, at least one participant worked in a managerial position.

\section{Log data}

The log data were gathered during workdays by utilizing the desktop logging application ManicTime (manictime.com 2017). The aim for the logging was to gain insight into workers' actions, work patterns, and applications used. As the log data were from the digital work environment, these data provide information about most tasks carried out by the participants. As the application contained reporting features for non-active sequences, the logging also accounted for breaks and other non-active sequences (e.g., meetings and telephone conversations). All participants were logged for one workday, and the mean duration of the log data was 7 hours 13 minutes.

\section{Workshop discussions}

Three workshops were held at each organization on their respective premises. Not all participants were able to attend all three workshops. The number of participants in individual workshops varied from 7 to 13 ; the average number of workshop participants was nine. The duration of each workshop was approximately 3 hours. The authors facilitated the group discussions by providing questions and themes for the participants to engage in. Participants were divided into two to three smaller groups in which they discussed the topics. In the discussions, participants reflected on the results of the questionnaire, log, and HRV measures presented by the authors. The participants discussed aspects of their work in the context of the digital work environment and wellbeing at work and reflected on how to reduce information load and promote information ergonomics. General discussions with all participants were also carried out to summarize the matters discussed. The facilitators were not present for the small-group discussions; the participants had the opportunity to discuss matters freely with their colleagues. However, the facilitators engaged in the summary discussions and could ask additional questions if needed. All group discussions were recorded and transcribed. The duration of the recorded data was 17 hours 41 minutes, and the length of the transcription 110,006 words.

\section{Analysis}

The log data analysis was automated by using certain scripts over the whole data set. In addition, task switches and communication-related actions were extracted from the data by manual classification. The outcome of the analysis was the time distribution between different applications and as derived from the use of applications for the respective task types. For the purposes of this paper, the most significant task type is the one related to communication. Each participant was analyzed according to the duration and 
proportion of communication-related tasks. Communication-related tasks were defined as the use of digital communication tools or channels.

Qualitative content analysis was applied to the workshop discussions. Content analysis was chosen for its flexibility in providing a data-driven approach for analyzing discussions and highlighting the central themes without preconceived notions from the sometimes scattered workshop data (Elo \& Kyngäs, 2008; Hsieh \& Shannon, 2005). The transcription of the discussions was read through. It became obvious after familiarization with the transcript that digital communication was one of the main factors associated with wellbeing in the digital work environment. After that, all the text covering communication in the digital work environment was highlighted. The transcription was read through again, and short notes to describe digital communication and its relation to wellbeing at work were written next to the text. Then, similar issues in the notes were identified, grouped together, and given descriptive preliminary names. Most issues came up repeatedly; the issues that came up very seldom were checked to see if they fit into the broader themes already recognized, and they mostly did. Single utterances that did not reoccur in the text and could not be placed in any of the emerging categories were excluded. The emerging categories were checked for similarities and merged together until six main themes were identified. The themes were the volume of digital communication, expectations of constant connectivity, the quality of the messages, adaptation of new tools, technical problems and negative user experience, and flexibility in communication.

\section{Results}

\section{The digital communication landscape}

Based on the log data, the communication environment of the participants was rich, providing them with several options for synchronous and asynchronous communication both internally and externally. The participants conducted most internal communication (except face-to-face) via digital channels. External communication was more restrained, with the participants expected to use formal means of communication, such as ticketing systems or team emails. However, the demand for practicality tended to overrule the instructions, and the participants often arranged communication according to personal preferences and habits.

The participants used various communication channels in their day-to-day work. The typical channels used included email, IM, ticketing tools, telephone, and Internet conference calls. Based on tracking and discussions, email was the main means of communication, and in most cases, the volume was significant. The average time spent engaged in email amounted to $7 \%$ of the workday. When other communication channels were added, the percentage of digital communication-related activities increased to $18 \%$. Most of the other communication was through IM or a ticketing tool. When all forms of communication that occur during working hours were included, $23 \%$ of working time was spent on communication-related activities, such as emailing, messaging, discussing, or engaging in meetings. The average duration that an email inbox was open was 15 minutes, but taking all options into account, the participants engaged in communication-related activity every 3 minutes, on average. 


\section{Perceptions of digital communication and its influence on wellbeing at work}

In the analysis of the workshop data, six themes emerged as the main digital communication factors affecting wellbeing at work. Five of the six themes constituted mostly demanding aspects of digital communication, while only one theme mostly described resources. The five themes covering mostly demands were the volume of digital communication, expectations of constant connectivity, the quality of the messages, the adaptation of new communication tools, and technical problems and negative user experiences. The resource category covered aspects of flexibility in communication. The six themes are discussed in more detail below.

The remarks made by participants during workshop discussions are marked with identifiers that include the field of the organization (e.g., insurance company), the participant number (p), the discussion group number (g), and the workshop number (ws). For example, the insurance company's participant number 2 in discussion group 3 in workshop 1 is identified as insurance company, p2, g3, ws1. The discussion group and workshop identifiers are provided because the assignment of participant numbers differed in each discussion group. In addition, participants could have different participant and group numbers in different workshops.

\section{The volume of digital communication}

The volume of digital communication and its effect on wellbeing were mostly addressed from two angles: the large quantity of messages and the variety of messages, which were also intertwined. In the workshop discussions, the participants noted that the increase in digital communication channels had made communications easier and thus increased the amount of communication. They discussed how colleagues and clients were reluctant to seek information themselves, because, for example, emailing or IM was perceived to be an easier way to obtain an answer. This behavior increased the amount of digital communication and the recipient's workload. The amount of digital communication was perceived to be extensive, especially the amount of email. The participants were often required to manage several inboxes, as they used team/group emails in addition to an individual work email. The team inboxes were described as extremely large and difficult to manage, as determining the intended recipient could be difficult: '[T]here's starting to be so much volume. And there's like five percent of the volume that concerns you' (industrial company, p3, g2, ws2). In addition, multiple email addresses increased the risk of messages being sent to the wrong address. Sometimes email messages became lost in the process, and the sorting took extra time and caused delays, especially if the misplaced email was important for a certain task.

In addition to the large quantity of email, the participants also mentioned the variety in the messages. Inboxes were filled with everything from advertisements to highly important information. A large portion of the mail was perceived to be irrelevant, and sometimes, it did not concern the recipient at all, largely because the messages were often sent to many recipients. The participants discussed how the employees and managers in their organizations often sent copies of email correspondence to a wide audience to reduce the chance of people missing information they might need or in which they might be interested: 
One of the issues regarding email that we were discussing previously is that email is sent in such large distribution. On the other hand, we want to distribute information easily to many, because someone could be like 'why didn't I get the information about something'. (insurance company, p2, g1, ws1)

However, the wide distribution of email usually generated even more unnecessary communication as the recipients often responded in equally wide distribution. This caused workflow interruptions and hindered concentration on tasks:

If you have a tight schedule for some task and you're concentrated and then email comes that has many recipients, and everyone's answering in turns and the answers come to everyone. It's distracting when it's bouncing back and forth because you always must check it in case there's something important. (financial administration services, p3, g2, ws2)

The volume of email was perceived to be so high that for many it was impossible to read everything. Some participants confessed to having thousands of unread messages: 'I have over 3,700 unread messages in my inbox' (industrial company, h3, g2, ws1). The piling up of email made it difficult to identify truly important and urgent messages from the less pressing ones. The participants felt perplexed about how to prevent important email from getting buried under the constant flow of messages. The participants had tried various methods for managing the incoming email, such as labeling and flagging, but with large quantities of messages, they often found it too bothersome. The participants reported feelings of anxiety because of the constant flow of messages. They often experienced the fear of missing something important and/or falling behind on their tasks. Replying as soon as possible was mentioned as a common way of keeping up. The participants said that they easily forgot about the messages if they did not respond right away, which resulted in replying to messages in various places and even while not at work:

With Outlook [...], if you don't do it right away, I don't know about you, but I fear I'm going to forget the thing, it gets buried because new [mail] is coming in all the time. Even if you flag it or something, but [...] how do you remember to come back to it? That's why you are checking it, and if there's that kind [of mail] that you feel you can give a quick response to, I get it done almost anywhere, I reply with my phone then. Then it's done and off the load. (industrial company, p3, g1, ws1)

The need to constantly keep up with the communication flow blurred the line between work and leisure time. Some participants discussed how they cleared their email in the evenings and weekends by replying to easier questions or deleting older mail. The participants felt doing this helped them to manage their work-related communication load and thus, their overall workload. However, checking email caused work to spill over into leisure time. A participant stated, 'One Sunday I had my work computer at home, and I was deleting some old email that had no function anymore, because there's like a thousand of those' (insurance company, p2, g2, ws2). Another participant said, 'I had done what I typically do at home: respond to email, pretty fast-paced, I put things forward' (financial administration services, p4, g1, ws1). 
In addition to email, participants discussed the constant flow of instant messages. Some participants said they often had multiple discussions open in IM at the same time:

I had an atypical day, because I didn't have four or five discussions going on in Skype simultaneously. It's pretty typical for me to have parts of the day when I have multiple conversations going on in there simultaneously. (financial administration services, p1, g3, ws1)

Some participants confessed they used IM even during meetings, which resulted in multitasking and dividing their attention among multiple separate discussions. The participants also stated that the communication coming through various channels sometimes overlapped; for example, the same information was sent via email and IM. The participants noted that there was a lack of shared practices for which communication channels to use and how to best use them. This resulted in the overlapping use of communication channels and increased the communication load.

\section{Expectations of constant connectivity}

Discussions about expectations of constant connectivity mostly covered two categories of expectation, explicit and implicit. Explicit expectations had to do with, for example, set response times and digital communication being a medium for assigning tasks. Implicit expectations, in contrast, were less direct and had to do with subtle or not-so-subtle ways of communicating the expectation of connectivity or a quick response.

Participants stated that the increase in digital communication had expanded the expectations for constant connectivity in their work. They felt strong pressure to be available and respond immediately. For some, email was the main medium for task assignment, and therefore, they were obliged to check it all the time. As one participant put it, 'For me, [email] is always the starting point of all work. Every request comes there. If I'm not checking it, I'm kind of not even at work then because it's for everything' (industrial company, p2, g3, ws1).

The participants discussed how they had a responsibility to actively monitor email. In every organization, tasks required checking email. The participants who constantly checked their email described it as stress-inducing. Most of the participants noted that it was difficult for them to control when they were reading email; they mostly felt the need to check the messages as soon as the mail arrived because there could be an urgent request or essential information for work tasks:

Outlook is [...] the environment in which we spend most of our workday. We were discussing if it'd be possible to check email in batches, because our observation was that, apparently, we are checking it steadily and pretty often throughout the day, so would it be possible to check it for example once an hour. The trouble we found with that is that sometimes the messages we get are urgent and there's this expectation that they get answered right away. (industrial company, p1, g1, ws1)

Constantly checking email frequently interrupted and fragmented tasks. The participants also noted that the interruptions made it difficult to concentrate. The interruptions could also disturb schedules and make it difficult to control one's work. However, the 
participants who worked in managerial or specialized expert positions had more control over when they were available. Some stated that they could block almost all communications if they were working with something that required their undivided attention, and that people in their organization usually respected this decision. In contrast, the employees who worked in direct customer service had practically no control over when they were available. The participants described the constant connectivity in customer service as very stressful; the situation was worst for employees who were required to be available through various communication tools at the same time:

In a perfect world, you could do one task at a time. You wouldn't have to be on the phone and then there's chat, or you have unfinished email, and the chat's going on. You could concentrate on one thing, finish it at once, and start a new one. (insurance company, p1, g2, ws1)

The customer service employees usually had strictly set priorities and response times. For example, the insurance company employees were required to respond to customer chat messages within 15 seconds. During busy periods, participants found this exhausting, especially if only a few employees were working during the shift.

In addition to performing tasks that required constant connectivity, the participants felt an implicit pressure to react immediately. They perceived constant connectivity to be a part of that organizational culture. The pressure was often enforced by consecutive communication if the recipient did not reply immediately. For example, an email could soon be followed by an IM or telephone call: 'If you haven't replied in half an hour, someone will chat' (industrial company, p3, g2, ws1). The participants also noted that people were often used to being served right away, and this habit increased the pressure for employees to be available:

The [sales] representatives and such contact us quite a lot through Lync because they want [to get in touch] fast. And you pretty much have to check the email all the time because if you haven't responded to the representative in an hour, they are coming to you through the lines. Because $[\ldots]$ they want and are used to being served right away and not in ten minutes. (insurance company, p7, g2, ws1)

The pressure to respond immediately was also communicated by marking messages as urgent. However, messages that were not as pressing were also marked as urgent: 'It's not always taken into consideration if it's actually urgent. Because usually they're not really urgent matters' (financial administration services, p2, g1, ws1). In some organizations, marking messages as urgent had already become meaningless. In the industrial company, there was a new category for truly pressing matters: 'Nowadays, tickets are no longer urgent; they're super urgent if they need to be handled immediately. The scale's shifting, apparently' (industrial company, p3, g2, ws1).

In the workshops, the participants also revealed that their need for uninterrupted work was often not respected. Instant messages in particular were sent even when the participants had marked their status as busy, and the employees were, for example, on the phone or in a meeting: 'If you're on red, yellow, anything, people don't respect that. Even if it says "in a meeting” or something' (insurance company, p1, g3, ws1). The participants often found this lack of respect highly irritating. They said it took them more 
time to complete tasks if they were interrupted, and interruptions also increased the risk of mistakes:

The possibility of errors increases so much [...] compared to when you're away from the line for a while. Ok, there may be some client waiting, but you're doing the task at once, it takes you a certain amount of time compared to when you have to interrupt it and start again. That takes a lot more time [...]; it basically doubles the work. (insurance company, p1, g3, ws1)

In addition to implicit pressure, some participants wondered whether constantly checking messages was related to personal preferences. Some said they were intrigued to see what was in the mail and wondered whether they had the self-discipline to refrain from constantly checking their email. Some also speculated that they were being too considerate and providing too good a service by replying right away, suggesting that they might be promoting the expectation of constant connectivity: 'At least for me, one thing regarding the managing of email is that I'm too kind. And because it's usually a clientrelated message, I react a bit too swiftly' (insurance company, p1, g1, ws1). However, the participants also noted that if they did not answer immediately, the lack of response generated even more communication and thus, increased the employees' workload.

\section{The quality of messages}

The discussions on the quality of messages mainly focused on messages that the participants perceived to be poorly constructed. This discussion primarily addressed two aspects of message content: inadequate information and etiquette. The participants discussed the impact of the quality of messages on their work. The discussions most often concerned email and tickets sent through ticketing tools. The participants stated that the messages were, at times, insufficient or open to multiple interpretations. The messages could lack crucial information or be poorly constructed, such that even determining the point of the message was difficult. The messages often demanded action from the recipient, which was not possible with the inadequate information received. The difficulty in proceeding with the matter generated more communication, as the recipient had to contact the sender to ask for more information:

Some say it in thousand words and some in too few, what is the problem and what they want. Or, depending on the other person's communication skills, you either have to ask for more [information] or otherwise use your time figuring out what they want. (industrial company, p1, g3, ws1)

Inadequate information in the message often resulted in longer communication chains as employees sent follow-up messages to obtain all pertinent information. The participants perceived inadequate information increased their workload and induced stress. However, they perceived clearly constructed messages that included all necessary information as streamlining the work: 'All the information correctly and clearly; that way you don't have to ask additional questions, you get it done at once' (financial administration services, p3, g1, ws2).

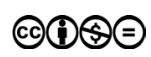


The participants wondered if the reasons for the poor quality of messages was that the messages were often read and written in a hurry. The participants also mentioned that some of the clients were not proficient in digital communication. Sometimes, the participants also thought the poor quality was due to a lack of manners. This lack of etiquette manifested, for example, in the use of excessive effects, such as writing in all caps (i.e., yelling) or using exclamation points and bold text: 'It's about etiquette, the use of the freaking exclamation points and all caps; you're like yelling in the messages' (industrial company, p2, g1, ws2). This behavior resulted in a loss of meaning, and the participants perceived it as annoying and sometimes even insulting.

\section{Adaptation of new communication tools}

In the workshops, the participants talked about the frequent need to adapt to new digital tools. The discussions were mostly divided into two aspects: the often inadequate resources for learning new technology and the increased workload caused by the addition of new tools. These new tools, intended to facilitate digital communication, were usually quite complex, such as collaboration tools that provided features for communicating and sharing but also for other purposes. Many felt pressure to keep up to date on new technologies but perceived the training to be lacking in their organizations. The participants often felt that they were left to their own devices when it came to learning new tools:

IT and SharePoints and such, the thing is that if we want to use them, there should be resources for the team and will from the management. And seriously, the calendar should be cleared, so that you have time to familiarize yourself. [...] I'm not staying here in the evening thinking about some SharePoint, what's SharePoint. First I Google 'what's SharePoint', and then I look at the IT pages [to see] if there are some instructions. Though I guess that's how it's supposed to be done. (industrial company, p5, g1, ws3)

The participants revealed that although instructions and training might be available in their organizations, no time was allotted. This lack of scheduling was perceived to be problematic, as the participants felt that they could not find the time needed for training. Thus, they tried to balance the, at times, contradictory-seeming needs of taking the time to learn new technology and performing their basic tasks. The participants found that trying to schedule training in the middle of all their other tasks was difficult and burdensome, and for some, it even induced feelings of guilt, as taking time for training meant that they left other tasks unattended:

Somehow, we could think [...] if we could take some study time, you would be allowed to put it in your calendar, you could arrange in your own group that there's a study hour. Because as soon as you take yourself away from the lines and you're not handling the mail, you get a feeling like... (insurance company, p1, g1, ws3) Yeah, like, 'I'm not making results, I'm not making decisions.' (insurance company, p4, g1, ws3)

The new communication tools were usually intended to replace email in certain cases. Although the participants liked the idea of receiving less email, they also pointed out 
that the new tools had no real benefit for them if they resulted in a similar or even larger workload:

The training and informing and supporting of a new practice, in the end we're talking about the same amount of work as if you handled it through email. So kind of, recognizing in what cases a tool could add genuine benefit, I think that's the challenge. (industrial company, p2, g1, ws3)

Some felt that they already had so many communication channels in use and such a large volume of messages that they had no capacity to add a new one: 'If at this moment you have trouble even having time to read the team inbox, so...' (industrial company, p2, g2, ws3). Participants also mentioned that adapting new communication tools required that the whole team be dedicated to learning and using the new tool, which often took more time and effort than sticking with familiar ones.

\section{Technical problems and negative user experience}

In the workshops, the participants described regularly encountering communicationrelated technical problems. The participants stressed the importance of functioning tools, as digital communication was an integral part of their daily work:

\footnotetext{
We both listed that Skype should work more reliably. It's such an important tool for us and there are at times challenges [...], there's some reason why it isn't always hundred percent foolproof. And then there are a lot of Skype meetings going on at the same time. So it's the kind of program that should definitely work when you need it. (financial administration services, p4, g1, ws1)
}

Application and network problems sometimes impeded digital communication. The participants reported experiencing delays in their work because of problems with communication tools. The delays caused by technical problems usually resulted in increased workloads after the tools started working again. At times, technical problems also prevented clients from getting in touch, which impeded customer service. In addition to causing delays, problems with communication tools could result in angry communication from clients:

We've had network problems quite a bit, and we're dependent on all the systems [...] then if they don't work [...] it frustrates and at least for me is a pretty hard stress-inducer. [...] And also you're just waiting when the clients come through the phone and are yelling and raging because nothing works. (insurance company, p1, g2, ws1)

In addition to the reliability problems with networks and communication tools, participants discussed the lack of user-friendliness of some communication tools:

We were talking about our [ticketing tool], how it's so rigid. And it's also a bit unclear how it looks to the customer when I'm answering a ticket [...] It's really messy and inflexible, the whole system. (financial administration services, p2, g1, ws1) 
The participants perceived some of the communication tools were insufficient for their intended purpose. The tools could be difficult to use, or they could lack the functionalities the participants perceived to be desirable. The participants thought these problems made work less efficient, and they caused frustration.

\section{Flexibility in communication}

The main positive attribute of digital communication described by the workshop participants was the flexibility it provided in their work. Flexibility was mainly discussed in the context of time, place, and situation or task. In addition to describing the downsides of digital communication, participants stated their belief that asynchronous communication was a big improvement over more traditional communication channels, especially the telephone. Participants mentioned telephone calls as the worst form of interruption, and for the most part, participants perceived the decreased number of phone calls positively. The participants noted that asynchronous communication was easier to manage. Even with the pressure to answer immediately, the participants felt that asynchronous communication, such as email and ticketing tools, provided them a better opportunity to decide when they were going to engage in communication activities: 'When it comes to time management, especially with email, [...] you're more of the master if you want to be' (industrial company, p3, g3, ws1). Another stated, 'Our [ticketing tool] makes it possible to plan like, okay, I'm not going to answer those today, I'll answer them tomorrow if the schedule allows' (financial administration services, p2, g1, ws2).

However, the participants also stated that some problems would be easier to resolve on the phone. A short phone call could answer a question that would require a long email chain to resolve:

The choice of a communication tool, like if you get a question in Lync or email, sometimes [...] it's better to just call. It takes couple of minutes, and the case is sorted, as opposed to when you often just write and write, and there's no end to it. (insurance company, p3, g1, ws1)

The participants also discussed their preference for variety in their communication channels. Some had established practices of what communication tools they used for what purposes. Participants preferred IM when they needed to ask something quickly: 'Sometimes, for example, if you're on a Lync meeting, and during the meeting you have to confirm something, then you can IM someone else' (insurance company, p2, g1, ws1).

The participants also described using different communication tools flexibly in chains; a case could be initiated quickly through IM, then the parties could work on their own, and later, they could follow up through email. Participants preferred email for more significant matters, as the messages themselves functioned as documentation: '[I] often use email to do summaries, as it leaves a document' (insurance company, p1, $\mathrm{g} 1$, ws1). The ease of documentation was mentioned as a simplifying feature in digital communication.

The participants noted that digital communication had made remote work possible, as the employees could access their email accounts almost anywhere with their phones. Teleconferencing also made it possible to attend meetings from home. However, 
the participants stated that they usually tried to attend meetings in person, as they valued face-to-face interaction, and they preferred to do more solitary work when they worked remotely: 'For me as a rule, if I have a meeting in my schedule, I'm not working remotely, even if I know that I can participate through Lync' (industrial company, p4, $\mathrm{g} 2$, ws2). Interestingly, although participants often found multitasking was burdensome, some stated that they liked to attend remote meetings and trainings because remote attendance made it possible to do other tasks simultaneously: 'What's good about [Lync] is that if there's some [...] good-to-know type of training, then I can listen and do other work on the side' (insurance company, p1, g1, ws1). This type of self-induced multitasking was not viewed negatively, while multitasking inflicted by an outside source was.

\section{Discussion and Conclusions}

The present study aimed to investigate the ways digital communication is associated with wellbeing at work. The study provides a novel contribution to the literature, as it adds to the limited number of studies focused on the digital communication environment as a broad phenomenon and provides a qualitative approach to the subject matter, whereas quantitative approaches have dominated in previous studies.

The results from the log data indicate a broad digital landscape with a large number of communication activities. A distinctive characteristic of the communication landscape was that most activities took place in digital form, such as through email, IM, or other digital means. The findings are in line with those of previous studies, which have also shown that the amount of communication has increased, and that, for example, the diversity of tasks has increased communication (see e.g. Mark et al., 2014; Okkonen et al., 2017). Understood in the context of previous studies, the present findings support the notion that employees work with multiple technologies at the same time and need to integrate and combine them effectively. Therefore, future studies should also investigate broad landscapes holistically rather than focusing on the use of single technologies.

In the analysis of the workshop discussions, it became obvious that digital communication affects wellbeing in various ways. Six themes were identified as the main factors of digital communication associated with wellbeing at work. The themes mostly represented demanding aspects. Only one theme, flexibility in communication, mostly highlighted digital communication as a resource that support employee wellbeing. This finding is in line with the findings of Stich et al. (2015), who identified four demands associated with ICT: response expectations, constant availability, increased workload, and poor communication.

The participants considered the volume of digital communication one of the most demanding aspects. This aspect included, for example, the problems of managing a large number of messages and maintaining multiple communication channels. To manage the amount of digital communication, the participants, for example, checked email after work. This result is in line with the results of previous studies (Barley et al., 2011; Brown et al., 2014; Diaz et al., 2012), which showed that a large quantity of digital communication is exhausting and often spills over from work to nonwork hours. This kind of spillover can be highly problematic, as it may induce unpaid overtime, impede recovery, and cause work-life conflict, which hinders employees' ability to detach from work and takes time away from nonwork activities.

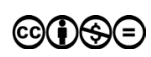


The participants also perceived expectations of constant connectivity as demanding. Constant connectivity included communication channels functioning as tools for task distribution, thus requiring constant monitoring and inducing a pressure to reply immediately. Previous research does not explicitly describe digital communication as a tool for task assignment, which the participants mentioned repeatedly. However, it has been noted that digital communication is an integral component of knowledge work, and the flow of digital communication cannot be separated from the workflow in general (Wajcman \& Rose, 2011). In addition, the expectation of availability and the implicit pressure to reply immediately brought up in this study have also been recognized as taxing features in previous studies (Barber \& Santuzzi, 2015; Barley et al., 2011; Brown et al., 2014; Wajcman $\&$ Rose, 2011). This study, along with previous studies, shows that the expectation of constant connectivity has multifold effects on the workflow and wellbeing of employees and that the demanding aspects of digital communication are interconnected and cannot be understood separately. For example, some of the previous studies identified the pressure to reply quickly as the reason for work-nonwork conflict (e.g., Barber \& Santuzzi, 2015), but in this study, the participants mainly discussed the spillover in the context of the large number of messages. However, this does not rule out the implicit expectations that may also lead this study's participants to check messages during nonwork hours.

The quality of messages had a negative effect on wellbeing, especially if the messages had inadequate information, which the participants perceived as increasing their workload. In addition, participants found messages that were poorly constructed or filled with effects frustrating. Previous studies (Brown et al., 2014; Day et al., 2010) also showed that digital communication quality (e.g., emotional or ambiguous content) may affect wellbeing at work. However, the main finding of this study regarding message quality-that inadequate information received in asynchronous communication generated more communication and increased workload-seems not to have occurred in other studies, although the notion of email ambiguity (Brown et al., 2014) may in part describes the same phenomenon.

Participants perceived that the adaptation of new communication technology often hindered wellbeing, as they perceived that the training provided was inadequate, and there was no time allocated for learning. This made it difficult to take time away from other tasks to learn new technology and even induced feelings of guilt when either the training or other tasks were left uncompleted. Previous research (Day et al., 2010; Salanova et al., 2014) also showed that the constant need to learn new technologies may increase work demands, anxiety, and stress. The situation is, in some ways, discordant, as new technologies are often intended to streamline communication flow (e.g., by reducing the amount of email). However, if employees are left to their own devices and the new tools are not introduced properly, they may simply add to the demands of an already complex digital work environment.

Participants also perceived that problems and negative user experiences with communication tools had negative effects on workflow. These problems included network problems hindering the use of communication tools and the tools' lack of user-friendliness. Previous research has shown that ICT problems, such as malfunctions and incompatible tools, induce strain (Day et al., 2010; Tarafdar et al., 2011). As digital communication constituted an integral part of the study participants' workflow, it seems self-evident that they brought up the technical aspect during the discussions. However, the participants mentioned the impact of technology functionality on wellbeing with considerably less frequency and emphasis than the impact of the volume of communication, expectations of 
connectivity, and message quality. This may indicate that the participants found that technology- and infrastructure-related factors were mostly sufficient and that the demanding aspects associated with digital communication were not due to the communication technology in itself but to the social factors related to digital communication, such as the organizations' and work teams' conventions and practices.

The one theme focusing mainly on resources supporting wellbeing at work was flexibility in communication, such as having different tools for different purposes, the possibility of scheduling communication activities, and the opportunity for remote work. Previous researchers (Barley et al., 2011; Day et al., 2010; Diaz et al., 2012; Mano \& Mesch, 2010) also showed that digital communication can provide flexibility but is also a burden. In this study, even with the perceived expectations of constant connectivity, the participants mostly preferred asynchronous communication because it provided them more ability to control and schedule the communication flow. In addition, the participants perceived possibility of remote work positively. When the participants saw flexibility in communication as supporting wellbeing, it mostly seemed to be related to an increased sense of control and autonomy over one's work. This perception indicates that although overwhelming associations of flexibility in digital communication (e.g., continuous flow of messages) may decrease employees' sense of control, flexibility can also help increase employees' control and autonomy, depending on the workplace's rules and practices.

The findings suggest that the demanding aspects of digital communication were more dominant. This finding is in line with the large body of research suggesting that users can experience ICT as demanding and stressful (e.g., Salanova et al., 2013, 2014; Tarafdar et al., 2011). There are many possible explanations for this finding. First, the current trend of digitalization may be translated into practice as a demand for the constant integration of new technology, which users typically experience as stressful (c.f. Tarafdar et al., 2011). Second, the data collection context may have influenced the results, as the aim of the project was to develop information ergonomics, not to simply collect data about the current state of affairs. This context made it more likely that participants would discuss problems that they felt needed to be fixed. Negativity bias, the common tendency to focus more on negative issues, may also explain the results in part (Rozin $\&$ Royzman, 2001). In addition, although the atmosphere of the discussion groups was constructive and open, the fact that the groups were organized at the workplaces likely influenced how the discussions proceeded. For example, experiences of demanding aspects of work can be quite personal, and employees want to present themselves as good and competent workers. Therefore, at least some social desirability bias might have appeared.

The practical implications of the study stem from its main findings-namely, that digital communication can function as a job demand or a job resource. The demanding aspects of digital communication can be either hindering or challenging, with varying implications for wellbeing at work. In general, it seems that digital communication acts as a resource when it increases a sense of control and autonomy over one's work. On the other hand, overwhelming and control-reducing aspects of digital communication seem to act as demands hindering wellbeing. Reflecting on the findings in terms of the previous discussion on information ergonomics, social factors seem to stand out more than other aspects of information ergonomics concerning digital communication and wellbeing at work. While some technology- and infrastructure-related factors came up, they did not seem to play as integral a role as social conventions and practices did. It could either be that technology- and infrastructure-related factors were found to be mostly

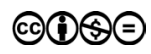


sufficient, or that these matters were seen as aspects that are out of the employees' control, and because of that they are not discussed in as much nuance or detail. Some individual-level aspects were also brought up, but they were still mostly connected to social aspects of information ergonomics-for example, how to manage one's workflow in certain types of organizational communication culture. In summary, it seems that social factors, such as organizational and team-level practices, have an important effect on communication-related wellbeing. The ways in which digital communication affect wellbeing at work often seem to be tied to these practices, which indicates that from the perspective of enhancing digital communication-related working conditions and wellbeing at work, greater leverage can be gained by developing well-communicated standard operating practices and conventions in the workplace.

\section{References}

Bakker,A. B. \& Demerouti, E. (2007). The job demands-resources model: state of the art, Journal of Managerial Psychology 22(3): 309-328. doi: https://doi.org/10.1108/02683940710733115.

Barber, L. K. \& Santuzzi, A. M. (2015). Please respond ASAP: workplace telepressure and employee recovery, Journal of Occupational Health Psychology 20(2): 172-189. doi: https:// doi.org/ 10.1037/a0038278.

Barley, S. R., Meyerson, D. E. \& Grodal, S. (2011). Email as a source and symbol of stress, Organization Science 22(4): 887-906. doi: https://doi.org/10.1287/orsc.1100.0573.

Brown, R., Duck, J. \& Jimmieson, N. (2014). E-mail in the workplace: the role of stress appraisals and normative response pressure in the relationship between e-mail stressors and employee strain, International Journal of Stress Management 21(4): 325-347. doi: https://doi.org/ 10.1037/a0037464.

Day, A., Scott, N. \& Kelloway, E. K. (2010). Information and communication technology: implications for job stress and employee well-being, in P. L. Perrewé \& D. C. Ganster (Eds.) New Developments in Theoretical and Conceptual Approaches to Job Stress: 317-350, Emerald. doi: https://doi.org/10.1108/s1479-3555(2010)0000008011.

Demerouti, E., Derks, D., Lieke, L. \& Bakker, A. B. (2014). New ways of working: Impact on working conditions, work-family balance, and well-being, in C. Korunka \& P. Hoonakker (Eds.) The Impact of ICT on Quality of Working Life: 123-141, Dordrecht: Springer. doi: https://doi.org/10.1007/978-94-017-8854-0 8 .

Diaz, I., Chiaburu, D. S., Zimmerman, R. D. \& Boswell, W. R. (2012). Communication technology: pros and cons of constant connection to work, Journal of Vocational Behavior 80(2): 500-508. doi: https://doi.org/10.1016/j.jvb.2011.08.007.

Elo, S. \& Kyngäs, H. (2008). The qualitative content analysis process, Journal of Advanced Nursing 62(1): 107-115. doi: https://doi.org/10.1111/j.1365-2648.2007.04569.x.

Eppler, M. J. \& Mengis, J. (2004). The concept of information overload. A review of literature from organization science, accounting, marketing, MIS and related disciplines, The Information Society 20(5): 325-344. doi: https://doi.org/10.1080/01972240490507974.

Franssila, H., Okkonen, J. \& Savolainen, R. (2016). Developing measures for information ergonomics in knowledge work, Ergonomics 59(3): 435-448. doi: https://doi.org/10.108 0/00140139.2015.1073795.

Hasle, P. \& Sørensen, O. (2013). Employees as individually and collectively acting subjects - key contributions from Nordic working life research, Nordic Journal of Working Life Studies 3(3): 9-30.

Hoonakker, P. (2014). Information and communication technology and quality of working life: Backgrounds, facts and figures, in C. Korunka \& P. Hoonakker (Eds.) The 
Impact of ICT on Quality of Working Life: 9-23. Dordrecht: Springer. doi: https://doi. org/10.1007/978-94-017-8854-0 8.

Hsien, H. F. \& Shannon, S. E. (2005). Three approaches to qualitative content analysis, Qualitative Health Research 15(9):1277-1288. doi: https://doi.org/10.1177/1049732305276687.

Mano, R. S. \& Mesch, G. S. (2010). E-mail characteristics, work performance and distress, Computers in Human Behavior 26(1): 61-69. doi: https://doi.org/10.1016/j. chb.2009.08.005.

Mark, G., Iqbal, S. T., Czerwinski, M. \& Johns, P. (2014). Bored Mondays and focused afternoons: the rhythm of attention and online activity in the workplace, CHI'14 Proceedings of the SIGCHI Conference on Human Factors in Computing Systems: 3025-3034. doi: https://doi.org/10.1145/2556288.2557204.

Mark, G., Voida, S. \& Cardello, A. V. (2012). 'A pace not dictated by electrons': an empirical study of work without email, CHI'12 Proceedings of the SIGCHI Conference on Human Factors in Computing Systems: 555-564. doi: https://doi.org/10.1145/2207676.2207754.

Okkonen, J., Heimonen, T., Savolainen, R. \& Turunen, M. (2017). Assessing Information Ergonomics in Work by Logging and Heart Rate Variability, Proceedings of the AHFE 2017 International Conference on Usability and User Experience: 425-436. doi: https://doi. org/10.1007/978-3-319-60492-3 41.

Orsila, R., Luukkaala, T., Manka, M. L. \& Nygård, C.-H. (2011). A new approach to measuring work-related well-being, International Journal of Occupational Safety and Ergonomics 17(4): 341-359. doi: https://doi.org/10.1080/10803548.2011.11076900.

Rozin, P. \& Royzman, E. B. (2001). Negativity bias, negativity dominance, and contagion, Personality and Social Psychology Review 5(4): 296-320. doi: https://doi.org/10.1207/ s15327957pspr0504 2.

Salanova, M., Llorens, S. \& Cifre, E. (2013). The dark side of technologies: Technostress among users of information and communication technologies. International Journal of Psychology 48(3): 422-436. doi: https://doi.org/10.1080/00207594.2012.680460.

Salanova, M., Llorens, S. \& Ventura, M. (2014). Technostress: the dark side of technologies, in C. Korunka \& P. Hoonakker (Eds.) The Impact of ICT on Quality of Working Life: 123-141, Dordrecht: Springer. doi: https://doi.org/10.1007/978-94-017-8854-0 8 .

Schaufeli, W. B. \& Bakker, A. B. (2004). Job demands, job resources, and their relationship with burnout and engagement: a multi-sample study, Journal of Organizational Behavior 25(3): 293-315. doi: https://doi.org/10.1002/job.248.

Schaufeli W. B. \& Taris T. W. (2014). A critical review of the job demands-resources model: implications for improving work and health, in G. F Bauer \& O. Hämmig (Eds.), Bridging Occupational, Organizational and Public Health - A Transdisciplinary Approach: 43-68. Dordrecht: Springer.

Somekh, B. (2006). Action Research: A Methodology for Change and Development, Maidenhead: Open University Press.

Stich, J. F., Farley, S., Cooper, C. \& Tarafdar, M. (2015). Information and communication technology demands: outcomes and interventions, Journal of Organizational Effectiveness: People and Performance 2(4): 327-345. doi: https://doi.org/10.1108/ LOEPP-09-2015-0031.

Su, N. M. \& Mark, G. (2008). Communication chains and multitasking, CHI'08 Proceedings of the SIGCHI Conference on Human Factors in Computing Systems: 83-92. doi: https:// doi.org/10.1145/1357054.1357069.

Tarafdar, M., Tu, Q., Ragu-Nathan, T. S. \& Ragu-Nathan, B. S. (2011). Crossing to the dark side: examining creators, outcomes, and inhibitors of technostress, Communications of the ACM 54(9): 113-120. doi: https://doi.org/10.1145/1995376.1995403.

Wajcman, J. \& Rose, E. (2011). Constant connectivity: rethinking interruptions at work, Organization Studies 32(7): 941-961. doi: https://doi.org/10.1177/0170840611410829. 